\title{
A New 30 GHz AMC/PRS RFID Reader Antenna with Circular Polarization
}

\author{
Omrane NECIBI, Chaouki GUESMI, Ali GHARSALLAH \\ UR13ES37 Research Unit for High \\ Frequency Electronic Circuits and Systems Tunis, \\ Dept. of Physics, \\ University of Tunis El Manar, \\ Faculty of Sciences of Tunis
}

\begin{abstract}
The work on this guideline focus on the development and the design of a circularly polarized metallic EBG antenna fed by two microstrip lines. In order to achieve that purpose, a list of indicative specifications has been established, namely, obtaining an antenna operating from 29.5 to $30 \mathrm{GHz}$, with a high gain value, an ellipticity rate less than $3 \mathrm{~dB}$ and a secondary lobes less than $-12 \mathrm{~dB}$ which is designed for Radio Frequency Identification (RFID) readers operating in the millimeter band. The size of the patch is $17 * 17 \mathrm{~mm} 2$. Artificial materials, such as artificial magnetic conductor (AMC) and Partially Reflective Surface (PRS) were added as an upper layer to this antenna in order to expand its bandwidth for the RFID reader applications. The new antenna has $\mathbf{- 3 5} \mathrm{dB}$ of insertion loss with an impedance bandwidth of $0.6 \mathrm{GHz}$ and a gain of $12.4 \mathrm{~dB}$ at $30 \mathrm{GHz}$. Analysis of the proposed antenna was carried out based on the finite element method using two electromagnetics simulations software: CST-MW Studio ${ }^{\circledR}$ and ANSYS HFSS. The simulation results obtained are presented and discussed.
\end{abstract}

Keywords-Radio Frequency Identification (RFID); FabryPerot Cavity Antenna (FPCA); Electromagnetics Band Gap (EBG); circular polarization; high impedance surface (HIS); artificial magnetic conductor (AMC); millimeter wave identification; Partially Reflective Surface (PRS); axial ratio (AR)

\section{INTRODUCTION}

The first developed RFID systems operated mainly at lowfrequency bands and have paved the way for the development of a new, more efficient and low cost RFID technology operating at higher frequencies: passive RFID in millimetre band.

Regardless of the antenna type, the aim of the majority of research studies was to improving of its performances, including a non-exhaustive list given below:

- Increase in operating frequency.

- Increase of the frequency band in adaptation, radiation and polarization.

- Increase in directivity.

- Increase in configurability antenna specifications (reconfigurable antenna in frequency, radiation pattern).

- Reduction of the congestion.

- Cost reduction.
All of these developments are of interest to the industrial community in order to meet the new demands of wireless communications.

Among the various research studies that were dedicated to the Electromagnetics Band Gap (EBG), antenna has caught our attention. Indeed, the latter is a good candidate to best meet some of these criteria and mainly, that of having a reduced thickness while the possibility of having a significant directivity.

Most of these antennas have linear polarization. However, the advantage of having a circular polarization is, in some applications, paramount or highly interesting. They are often used in satellite systems and radars, where the position of the transmitting and receiving antenna varies over time (vehicles, aircraft, etc.). In this type of communication, the antenna often sought is a circularly polarized antenna associated with a high gain. It was shown in the literature that a large number of sources (or antennas) making it possible to generate a circular polarization and capable of exciting the EBG antenna. Among these we can find the printed antenna [1]-[9] (or more commonly called patch antenna), the helical antenna, the slots [10] and fed cones [11] Waveguide and other 3D solutions. It should be noted that this list is not exhaustive and can be completed.

In this paper, the choice was focused on printed antennas because of their small size compared to the other structures mentioned previously. Their main advantage is that they maintain an important compactness of the EBG structure, which was evaluated at quantities less than one-tenth of the wavelength [12]-[15]. Moreover, this solution was attractive because of the simplicity of its implementation, its cost and its low weight.

The main techniques for generating a circular polarization with this type of aerial differ generally in the number of excitations that are considered: A simple excitation [1]-[5], a double excitation [6]-[9] and finally a quadruple excitation [16]-[17].

In general, for an antenna to generate a circular polarization, the orthogonal components (E $\theta$ and $E \Phi)$ of the electric field must have the same amplitude and must be in phase quadrature. In the case of the patch, this equi-amplitude is obtained by an optimization of the latter. Whilst the phase 
quadrature is carried out either by deforming the geometry of the radiating element in the case of a simple supply or by bringing an external device to the patch of the coupler or distribution type in the case of a double and a quadruple excitation. It should be noted that the first two solutions (single and double excitation) are more common than the latter. As mentioned previously, this type of polarization was widely used in spatial communications because it increases the robustness of the system considered against the various disturbances associated with wave propagation (depolarization, reflection, etc.). In most cases, antennas designed for this purpose are developed only to generate a good circular polarization in a given direction or pointing to its closest neighbors.

The study will be limited to a dual-source antenna. As a result, the work presented in this paper is entirely dedicated to the design of circularly polarized metal EBG antennas fed by two microstrip lines in order to have an antenna operating between 29.5 and $30 \mathrm{GHz}$, with a high gain, an ellipticity of less than $3 \mathrm{~dB}$ and secondary lobes of less than $-12 \mathrm{~dB}$.

\section{ANTENNA DESIGN}

The used methods to obtain the circular polarization with the printed antennas are generally divided into two categories. The first uses two power sources (probe, slot ...) separated spatially and temporally by $90{ }^{\circ}$.The use of an external polarizer, such as a Y junction or a hybrid coupler, is crucial. The second uses a single source power with disturbing elements such as slots, truncations, and pins in order to create two orthogonal modes linearly polarized and excited in quadrature phase. Here are some examples of these different methods. First, let us see the first process.

\section{A. Patch with a Dual Feed Probe}

The simplest method is to use two probes positioned orthogonally on the circular or square patch [1]. It is fed by two electric fields of the same amplitude and in phase quadrature (Fig. 1). However, this type of antenna is limited in the operating band because of its adaptation band (2\%) while that of the axial ratio is still high.
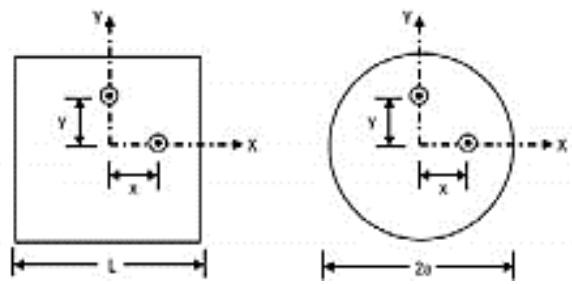

Fig. 1. Different patch configurations powered by two probes.

Several techniques can improve the performance of this type of antenna. The first is to add additional probes [1]. The second is to add additional patches positioned next to the main patch [18]. The third is to add a parasitic "patch" over the main patch [19]. The probes can be replaced by slots, allowing coupling feeding, but this technique is not very efficient [20]. Special shapes of slits can be used to improve the specifications [21]. All these antennas need an external element to feed them properly. This device entails an additional cost and generates imperfections that deteriorate the circular polarization. There is another method for this type of aerial namely a single power supply with the interferers.

\section{B. Patch Antenna Fed by a Microstrip Line}

In order to use a microstrip line instead of a coaxial cable to power the antenna, two different approaches can be applied. One method is to connect directly a characteristic impedance line $50 \Omega$ to the antenna (Fig. 2). In this case, the microstrip line should be connected to the antenna using two notches and should be matched to $50 \Omega$ of the transmission line [22], [23].

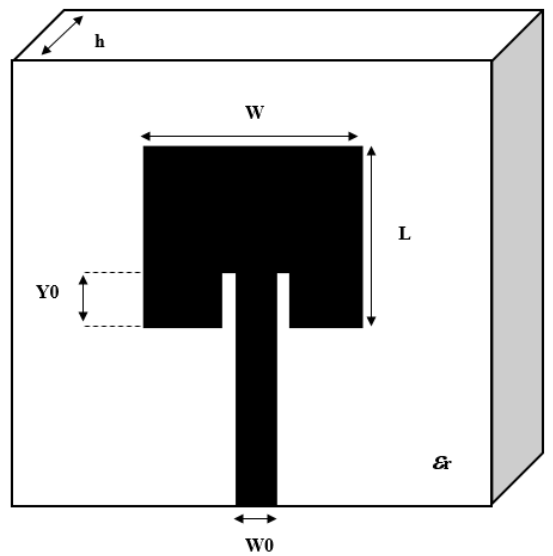

Fig. 2. Patch antenna powered directly by microstrip line.

Another method is to connect the microstrip line to the edge of the antenna (Fig. 3). In this case, the quarter wave transformer must be placed between the microstrip line and the antenna for the $50 \Omega$ impedance assortment.

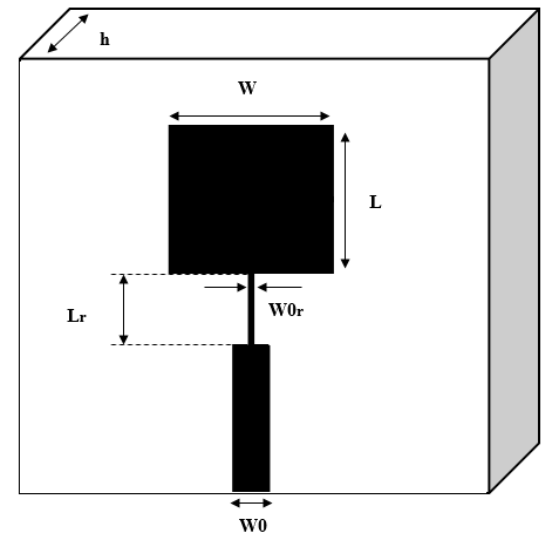

Fig. 3. Patch antenna powered by microstrip line using the quarter-wave transformer.

For both methods, the microstrip line width of characteristic impedance $50 \Omega$ should be calculated.

The characteristic impedance of a quarter-wave transmitter is [24]:

$$
Z_{r}=\sqrt{Z_{c} Z_{L}}
$$

Where, $Z_{c}$ is the characteristic impedance of the microstrip line, $Z_{L}$ is the patch edge impedance.

The characteristic impedance of a microstrip line is: 
$Z_{c}=\left\{\begin{array}{c}\frac{60}{\sqrt{\varepsilon_{\text {reff }}}} \ln \left(\frac{8 h}{w_{0}}+\frac{w_{0}}{4 h}\right) \\ \sqrt{\varepsilon_{\text {reff }}}\left(\frac{w_{0}}{h}+1.393+0.667 \lambda v\left(\frac{w_{0}}{h}+1.444\right)\right)\end{array}\right.$

Where, $w_{0}$ is the width of the microstrip line.

$\mathrm{h}$ : the thickness of the dielectric substrate.

$\varepsilon_{\text {reff }}$ : effective permittivity of the substrate.

$$
\varepsilon_{\text {reff }}=\frac{\left(\varepsilon_{r}+1\right)}{2}+\frac{\left(\varepsilon_{r}-1\right)}{2}\left(1+12 \frac{w}{h}\right)^{-1 / 2}
$$

Alternatively, using the following equation (if $w_{0}$ is not available), to be used as the first input for an iterative design:

$$
\varepsilon_{\text {reff }} \simeq \frac{\left(\varepsilon_{r}+1\right)}{2}
$$

Finally, the length of the transformer can be calculated by:

$$
\lambda_{r}=\frac{\lambda}{4}=\frac{\lambda_{0}}{4 \sqrt{\varepsilon_{\text {reff }}}}
$$

Impedance matching is a very important operation in the supply of an antenna, whatever the technique used, since it ensures that most of the power is transferred from the power supply to the radiating element, that is to say the patch. In addition to impedance matching, there is parasitic radiation and surface wave losses that are caused by discontinuities such as fitting elbow fittings and impedance transformers.

\section{Analysis and Simulation Results} Fig. 4.

The geometry of the single antenna element is shown in

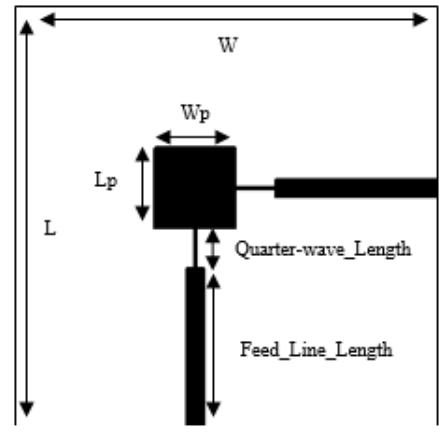

Fig. 4. Geometry of the proposed antenna, (x-y plan).

The physical dimensions of this antenna are shown in Table 1

TABLE I. ANTENNA DIMENSIONS

\begin{tabular}{|l|l|l|l|l|l|l|}
\hline Parameters & L & W & Lp & Wp & $\begin{array}{l}\text { Feed_Line } \\
\text { _Length }\end{array}$ & $\begin{array}{l}\text { Quarter- } \\
\text { wave_Length }\end{array}$ \\
\hline $\begin{array}{l}\text { Dimensions } \\
(\mathbf{m m})\end{array}$ & 17 & 17 & 3.2 & 3.2 & 6.45 & 1.52 \\
\hline
\end{tabular}

The value of standing wave ratio, VSWR, serves a good measure of whether the system is working effectively (Fig. 5).
In the present work, the simulated VSWR value is well below 2 in the frequency band.

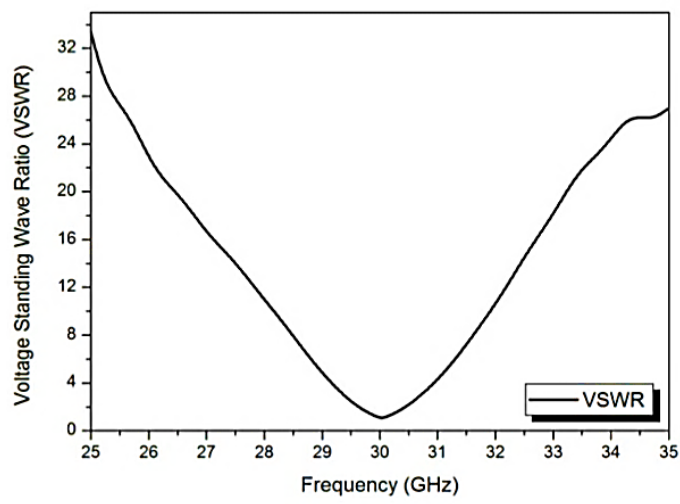

Fig. 5. VSWR.

Several articles define the antenna band use for $|\mathrm{S} 11|<-10$ $\mathrm{dB}$, and others for $|\mathrm{S} 11|<-15 \mathrm{~dB}$ or $|\mathrm{S} 11|<-20 \mathrm{~dB}$ [25], [26]. This paper work with the first definition $|\mathrm{S} 11|<-10 \mathrm{~dB}$ : the band of use ranges from 29.5 to $30.5 \mathrm{GHz}$, the antenna has a bandwidth of about $2.77 \%$ around the frequency centre.

The curves are quite consistent and have similar shape with a frequency shift rate equal to $1 \%$. The difference observed coming from the antenna because the reflection coefficients of the latter are shifted frequently between CST MWS Software ANSYS HFSS Software (Fig. 6).

This difference comes from the difference in solving the Maxwell equations between the two methods. However, the two methods are consistent with our objectives as the S11 module is less than $-10 \mathrm{~dB}$ on the work tape.

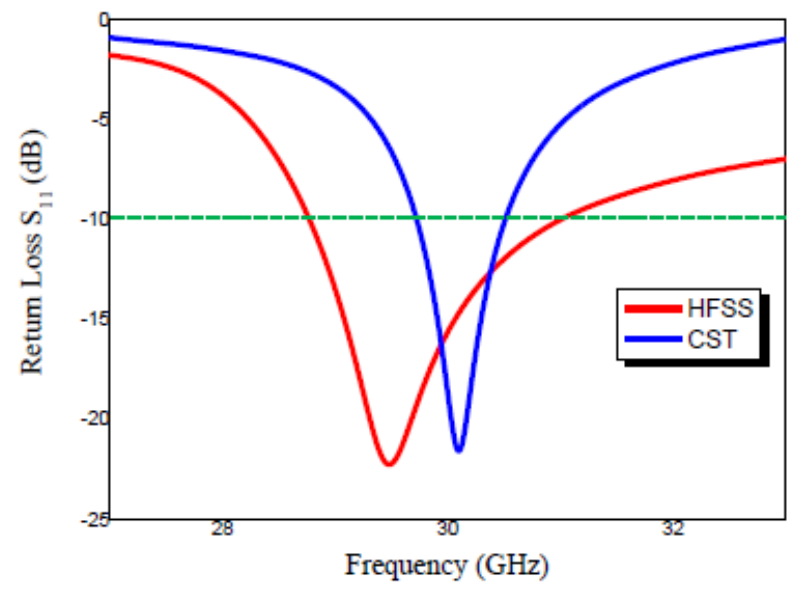

Fig. 6. Simulated reflection coefficient for the proposed antenna.

Antenna simulation results in terms axial ratio, are shown in Fig. 7(a) and (b).

The antenna is fed by two lines that have a characteristic impedance of $50 \Omega$. The axial ratio remains below $3 \mathrm{~dB}$ between 29.5 and $30.5 \mathrm{GHz}$. Due to the symmetrical shape of the antenna, only the results for one of the ports are presented. 


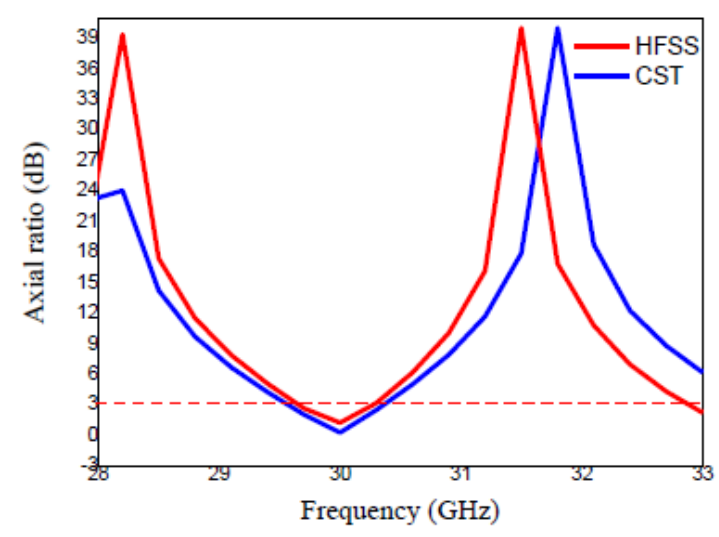

(a)

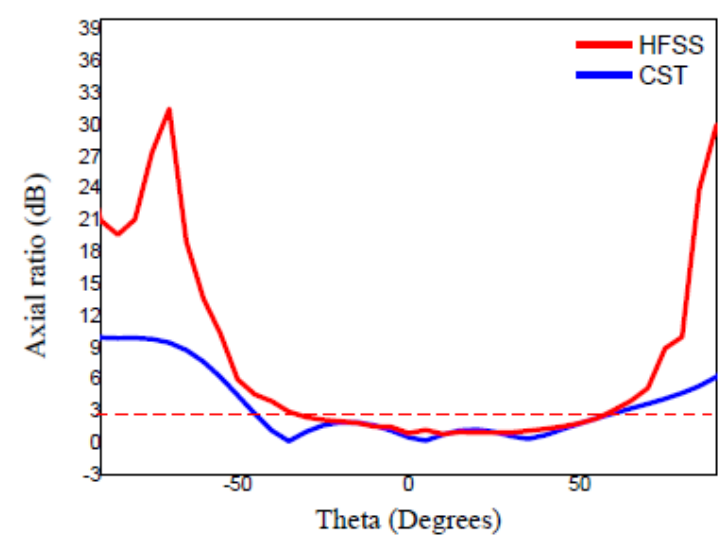

(b)

Fig. 7. Axial ratio as a function of (a) frequency, (b) elevation.

The value of the simulated gain in circular polarization of this antenna is $8.16 \mathrm{~dB}$. Fig. 8 shows the gain pattern in polar coordinate.

Farfield Realized Gain Abs (Phi=0)

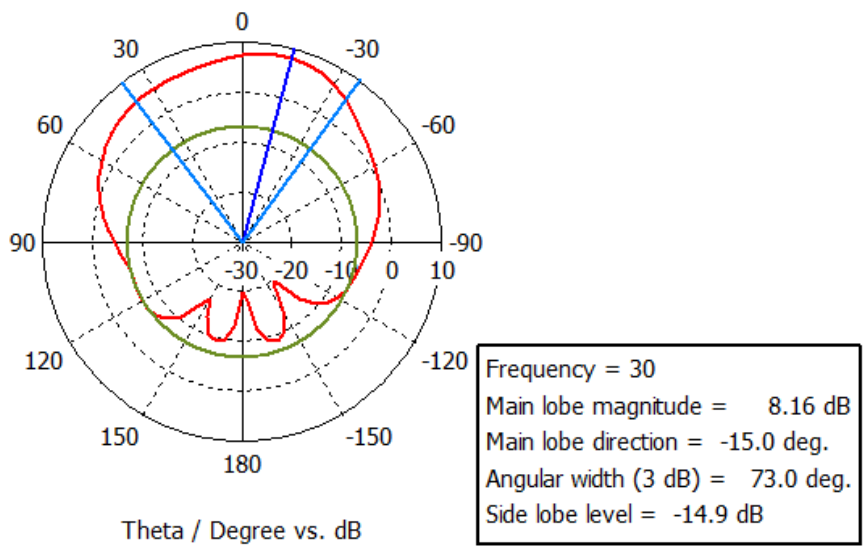

Fig. 8. Radiation pattern of proposed antenna (30 GHz) (CST MW Result simulation).

\section{EBG ANTENNA DESIGN}

We will now discuss the gain and the directivity of the antenna and the influence of high impedance surface on these two parameters.

\section{A. AMC Unit Cell}

High Impedance Surface (HIS) structures, also designed as Artificial Magnetic Conductor (AMC) or Perfect Magnetic Conductor (PMC) [27], [29] may be very useful for antenna applications and in a very large variety of microwave other devices [28], [30].

Fig. 9 illustrates the orientation of the metamaterial unit cell in the CST MWS Studio simulation setup. The perfectly electrically conducting and perfectly magnetically conducting boundary conditions were defined in the $\mathrm{x}$ and $\mathrm{y}$ directions in the simulation setup, and the structure is excited by a uniform plane wave propagating in the $\mathrm{z}$ direction.

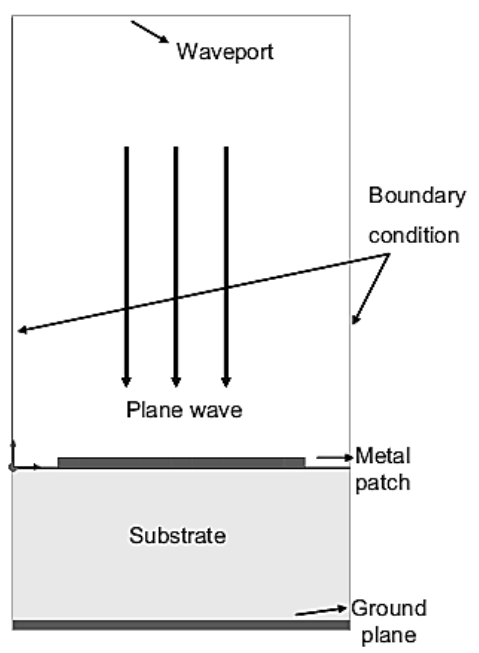

Fig. 9. Setup simulation for one cell.

The AMC condition is characterized by a resonance frequency where the phase of the reflection coefficient is zero and its magnitude equal to one.

The geometry of the proposed AMC shown in Fig. 10 is constituted of a dielectric layer (Rogers RT5880, $\varepsilon r=2.2$ and loss tangent equal to 0.0009 ) with a height of $0.254 \mathrm{~mm}$ containing the ground plane on its lower part (visible in Fig. 10(b)). It is printed on both sides with a copper thickness of 2 microns.

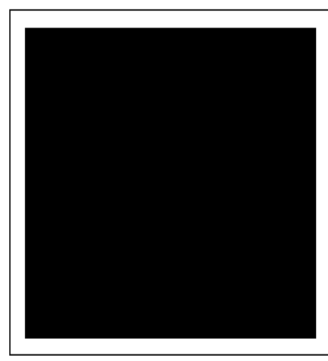

(a)

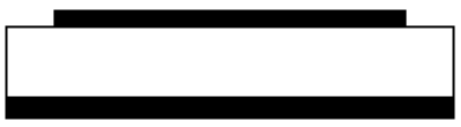

(b)
Fig. 10. AMC Unit cell geometry, (a) Front view, (b) bottom view. 
This structure is achievable with industrial manufacturing processes based on dielectric monolayers. We shall now turn to its electromagnetic properties.

\section{B. Numerical Characterization}

The properties of this structure were presented under normal incidence using a classical calculation on an elementary cell. Then its resonant frequency was determined, allowing defining the size of the elementary cell depending on the wavelength.

The study of an elementary cell allowed us to observe the properties of an infinite plane made up of periodic AMC elementary cells [31], [32]. By taking advantage of the periodicity and symmetry properties of these structures, the phase of the reflection coefficient can be easily calculated under normal incidence. The surface impedance characterization device uses a guide consisting of perfect electrical conductors (PEC) and alternate perfect magnetic conductors (PMC) (Fig. 11). Thus, a TEM wave is propagated in this guide terminated by the elementary AMC cell. The study of the reflection properties of this mode is equivalent to the study of the reflection properties of a planar wave on an infinite plan constituted by AMC periodized under normal incidence. The electromagnetic simulations were carried out with the CST MWV studio.

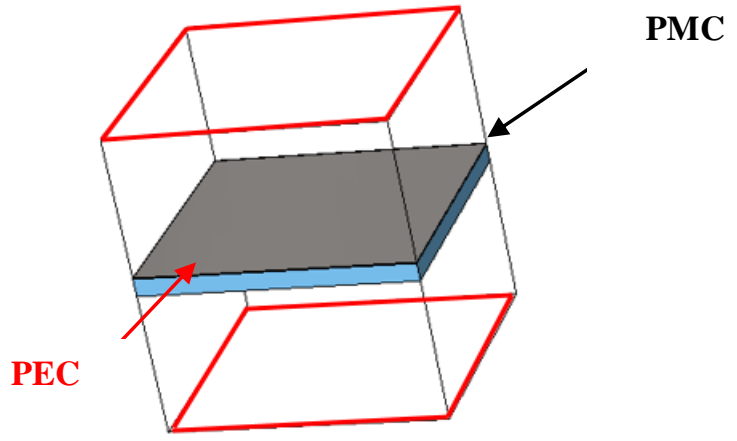

Fig. 11. 3D view of the device for characterization of an infinite plan of AMC patterns under normal incidence.

\section{Reflection Phase at $0^{\circ}$}

From such an electromagnetic calculation, the phase of the input port is estimated. Knowing the propagation properties of the wave in the waveguide, it is then possible to know the phase of the reflection coefficient at the top of the high impedance surface.

The resonance of the structure is characterized by a zero phase shift at reflection (Fig. 12). Thus, we observe at the resonance point a reflection coefficient close to that of a perfect magnetic conductor $(\Gamma=+1)$, which has an infinite surface impedance and a zero phase shift at reflection for all frequencies.
By defining the bandwidth HIS by the frequency band, having a zero phase of reflection, between $-45^{\circ}$ and $+45^{\circ}$, the HIS operates between $29.1 \mathrm{GHz}$ and $30.9 \mathrm{GHz}$ (Fig.12). The associated bandwidth is thus $4.67 \%$ with a peak resonance at $30 \mathrm{GHz}$.

Fig. 12 shows the phase diagram of a simulated AMC using temporal solver from CST MWS for a normal incidence.

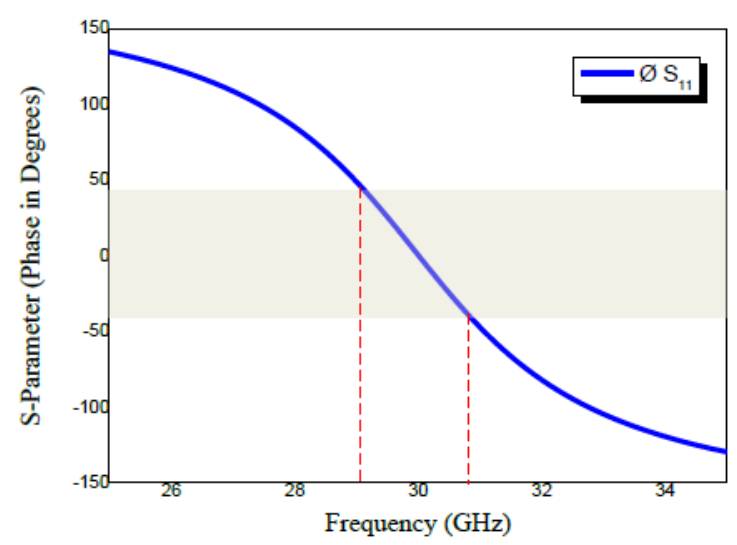

Fig. 12. Reflection phase variation of the AMC versus frequency (CST MW result simulation)

Using this characterizing technique of the surface impedance, it was possible to adjust the resonance frequency of the structure by playing of certain parameters. Indeed, it was known that this resonance results from the combination of inductive and capacitive effects [31]-[34]. To the extent that we know the origin of these effects - the volume of the substrate of the elementary AMC cell for the inductive effect and the surface of the patches opposite for the capacitive effect - we can adjust the size of the patches and on the volume of the substrate to tune the resonance frequency. From the simple circuit modelling, taking into account the phenomena involved [31], it was sufficient to increase the value of the capacitive effects (by increasing the surface area of the upper and lower patches opposite) or inductive (by increasing the volume of the AMC substrate) to lower the resonant frequency, the latter being proportional to $1 / \sqrt{L / C}$. On the other hand, the bandwidth associated with this resonance varies proportionally with $\sqrt{L / C}$. Thus, we can increase the bandwidth by favouring the inductive effects in relation to the capacitive effects.

The size of the AMC cell designed is $3.7 \mathrm{~mm}$ by $3.7 \mathrm{~mm}$. When compared to the wavelength of the AMC resonance frequency, the elementary cell has a size of $0.37 \lambda * 0.37 \lambda$.

\section{$D$. Influence of the AMC Layers Surrounding the Antenna}

Fig. 13 indicates that there was a propagation of the surface waves on the antenna plane until they reach the edges where they can radiate into free space; these waves are represented as undulations in the radiation pattern. 


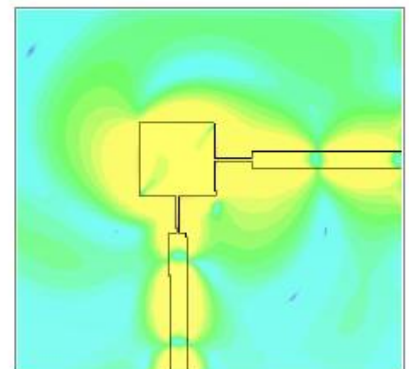

(a)

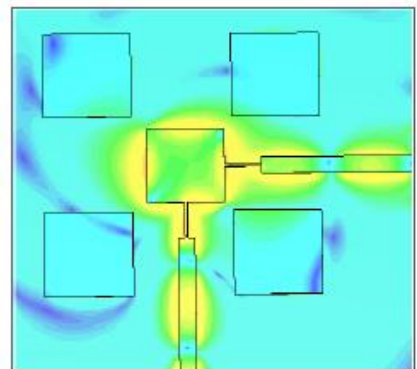

(b)
Fig. 13. Propagation of surface waves on the antenna (a) without AMC, (b) surrounded by four cell of AMC. (CST MW Results simulations).

However, when adding a layer of AMC, surface waves will not reach the edges of the surface below the patch. These waves were not visible through the cobblestones which act as an obstacle and remove these waves (filtering property), which explains the disappearance of the undulations in the field pattern.

\section{E. Results}

The good level obtained by the numerical results, allows us to conclude that the models are adequate to quickly size of HIS for use as metal barriers, such as absorbent surfaces or reflector plan in the proposed antenna.

The S11 of this antenna is shown in Fig. 14.

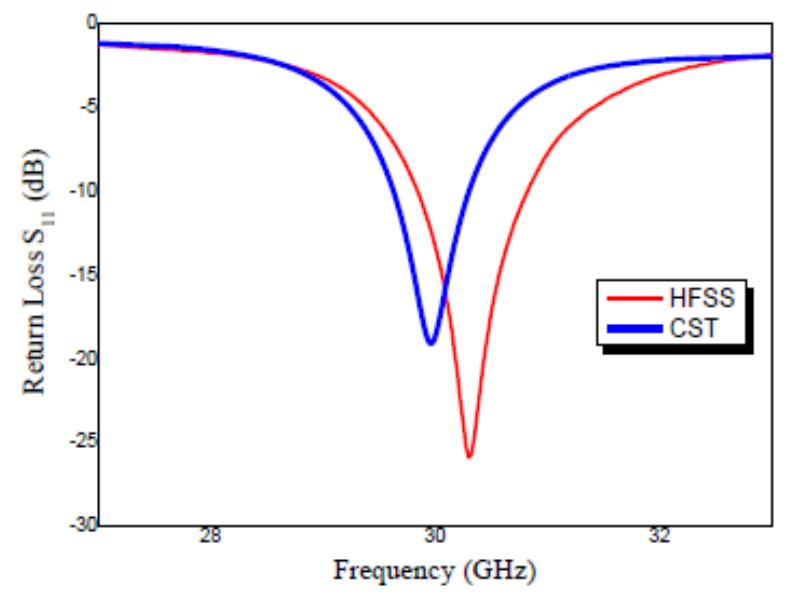

Fig. 14. Simulated reflection coefficient for the proposed antenna with AMC.

Even when the AMC has been added, it is still noticeable that there is an axial ratio of less than $3 \mathrm{~dB}$, which confirms that the performance of the basic antenna has been improved while maintaining the circular polarization.

Axial ratio simulation results of this antenna are shown in Fig. 15(a) and (b).

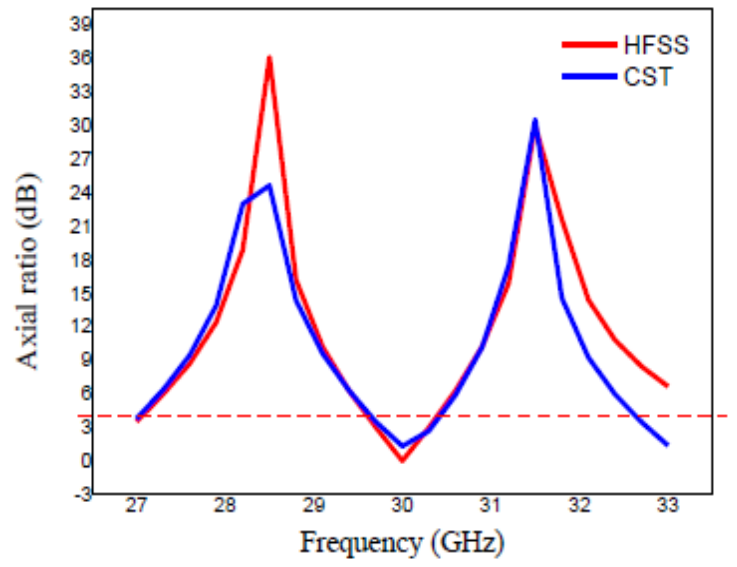

(a)

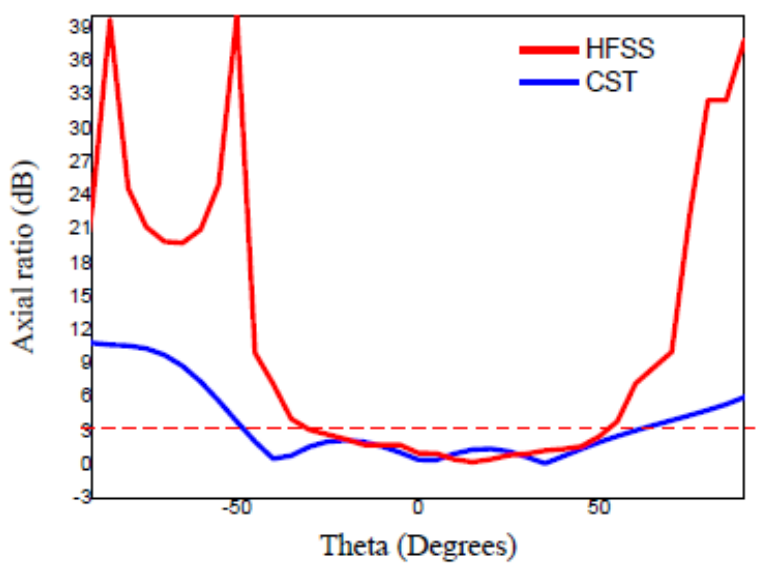

(b)

Fig. 15. Axial ratio as a function of (a) frequency, (b) elevation.

A slight enhancement of more than $1 \mathrm{~dB}$ was achieved in the gain relative to the antenna that is depicted in Fig. 8 while maintaining a very low side lobe level (less than $-12 \mathrm{~dB}$ ) equal to $-16.5 \mathrm{~dB}$. The gain obtained is $9.17 \mathrm{~dB}$. Fig. 16 shows the gain pattern in polar coordinate.

$$
\text { Farfield Directivity Abs (Phi=0) }
$$

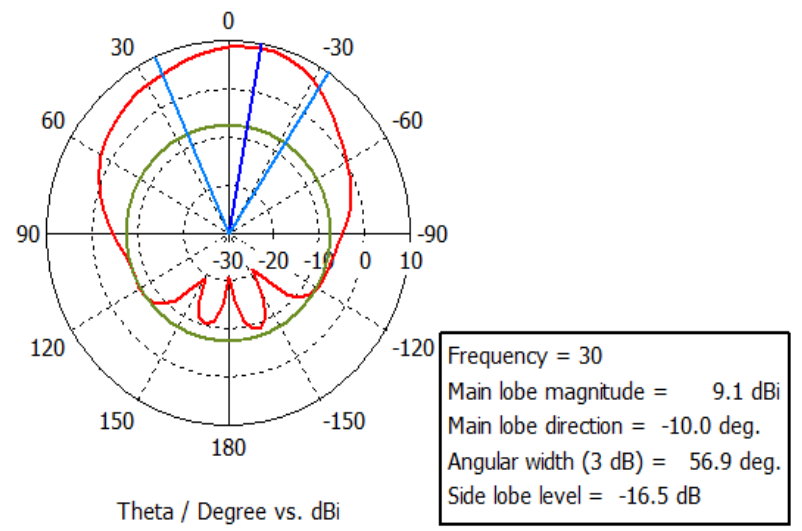

Fig. 16. Radiation pattern of proposed antenna with AMC (CST MW result simulation). 


\section{PRS ANTENNA DESIGN}

The height of the EBG antenna depends on the reflection properties of the used EBG material (generally consisting of dielectric plates and metal rods). Indeed, the phase of the reflection of the latter, which tends towards $\pi$ [35], imposes a resonance close to $\lambda / 2$.

$$
h=\frac{\lambda}{2} *\left(\frac{\phi_{\text {EBGmaterial }}+\phi_{\text {Graoundplan }}}{2 * \pi}\right) \approx \frac{\lambda}{2}
$$

During the last decade, several studies [36]-[38] have been carried out to reduce the height of the EBG resonator. Their common feature was the use of the HIS, the most known structure of which was called mushroom and was developed by D. Sievenpiper [39]. It consists of rectangular (or square) patterns arranged periodically above a ground plane and which are short-circuited to it. However, the HIS can also be used without the short-circuiting pads [38].

In both cases, this type of structure is defined in the literature as PMC (Perfect Magnetic Conductor), which has the advantage of transforming the frozen reflection properties of the ground plane $(\pi)$ into a Magnetic Short Circuit (MSC) with a phase equal to $0^{\circ}$ at a given frequency. These structures behave like AMC on a frequency band where the phase is not perfectly equal to 0 .

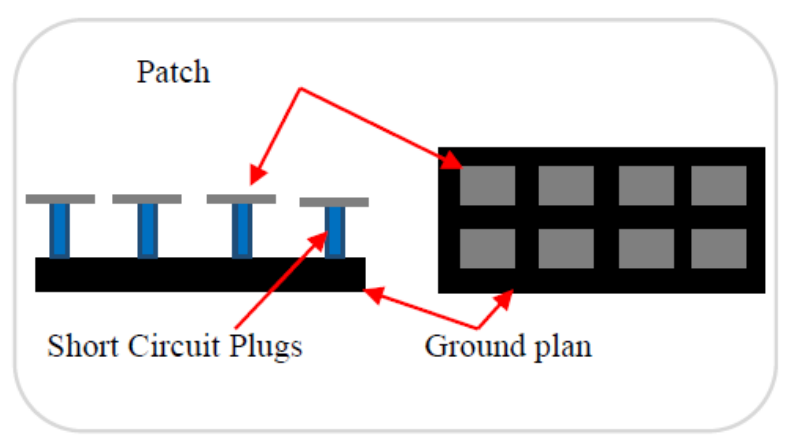

(a)

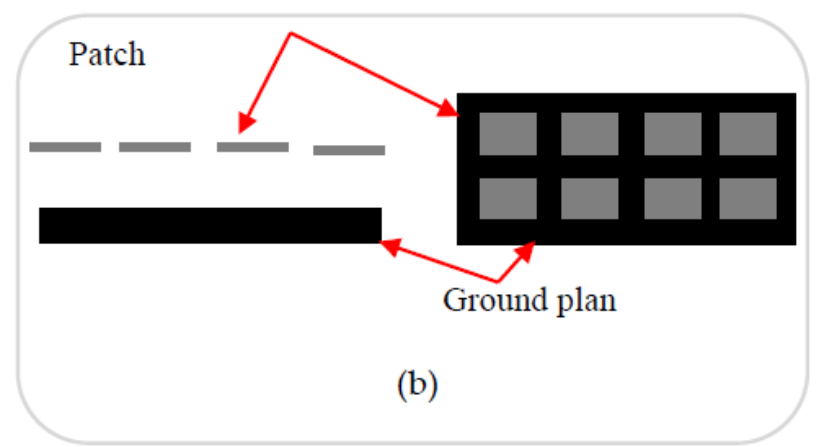

Fig. 17. Illustration of AMC structures, including the Sievenpiper mushroom, (a) with short-circuit pads, (b) without short-circuit pads.

By using an AMC having a reflection phase equal to $0^{\circ}$ (Fig. 17) as the ground plan of an EBG antenna, the resonance conditions are then modified, thus making it possible to position the EBG material to either $\lambda / 2$ as was the case in the presence of a standard metal ground plane, but at $\lambda / 4$.

$$
h=\frac{\lambda}{2} *\left(\frac{\phi_{E B G m a t e r i a l}+\phi_{A M C}}{2 * \pi}\right) \approx \frac{\lambda}{4}
$$

Using this principle, a gain of $19 \mathrm{~dB}$ was obtained and the bandwidth set at $-3 \mathrm{~dB}$ of the maximum is $2 \%$ [37].

It is possible to further reduce the height of the EBG resonator if the AMC structure is used in the band where the phase of reflection is close to $-\pi$ (Fig. 18) [38]. A resonance for height $h$ less than $\lambda / 10$ is then possible. This results in very compact antennas.

$$
\lim _{\phi_{A M C} \rightarrow-\pi}(h \ll \lambda)
$$

This evolution of the compactness of the EBG antenna by using the AMC structure is illustrated below (Fig. 18):

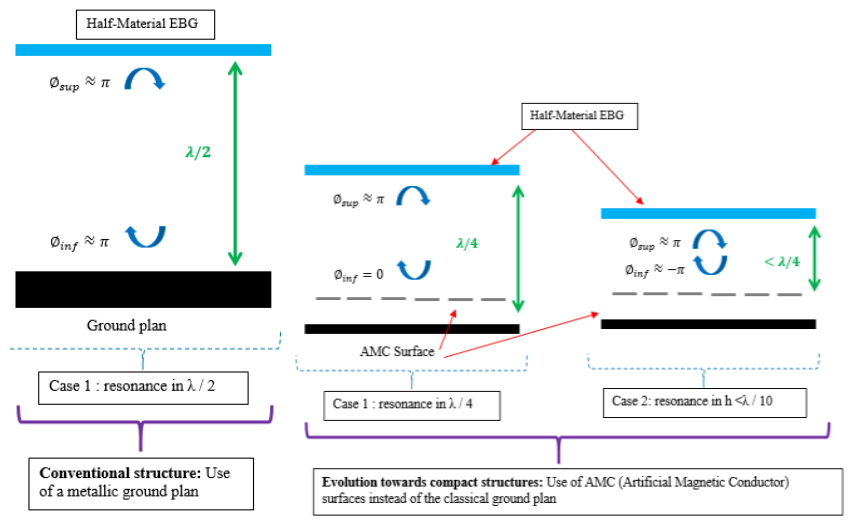

Fig. 18. Evolution from conventional EBG antenna to more compact structures [40].

To conclude on this study, it should be noted that the height reduction of the EBG resonator is obtained with AMC structures, which, due to their varying phase of reflection, make it possible to modify the properties of the ground plan and thus lead to Very compact structures. However, these structures have the disadvantage of being multilayer and difficult to realize.

This study is conducted in order to improve the directivity in the cavities based on metamaterials. Fig. 19 shows the unit cell of the PRS based metamaterials used as a reflector. It is composed of a capacitive grid on one face of the substrate Rogers Duroid 5880 having a thickness $\mathrm{d}=0.127 \mathrm{~mm}$ and a permittivity $\varepsilon_{r}=2.2$ and an inductive grid on the other side. The capacitive and inductive gates are respectively constituted of a network 2-D circular pellet and copper crossed ribbons.

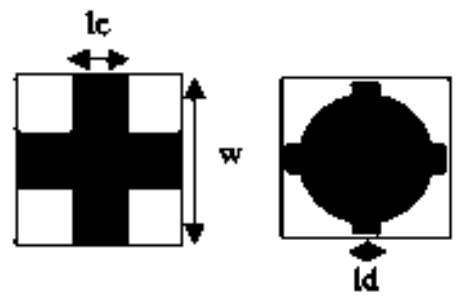

Fig. 19. The unit cell of the PRS structure, where $1 \mathrm{c}=1.2, \mathrm{w}=3.7, \mathrm{ld}=0.64$ (all dimensions in $\mathrm{mm}$ ). 


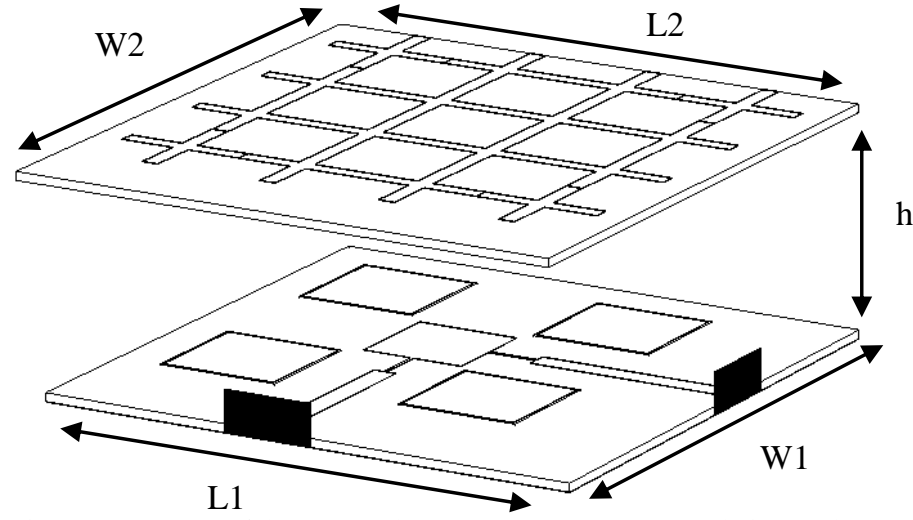

Fig. 20. Geometry of the proposed PRS-AMC-antenna (General view), where $\mathrm{L} 1=17, \mathrm{~W} 1=17, \mathrm{~L} 2=17, \mathrm{~W} 2=18$ (all dimensions are in $\mathrm{mm}$ ).

The cavity model was used to estimate the transmission and reflection of the PRS under normal incidence. It was the first step that permitted the reduction of computational time and memory. Then, the periodic structure PRS is placed above the antenna at estimated height, as illustrated in Fig. 20. The structure has been simulated using time domain solver of CSTMW studio.

\section{F. Antenna Performance}

CST software is used to simulate the PRS structure and to obtain its phase and magnitude reflection coefficient $\left(\boldsymbol{S}_{11}\right)$.

Fig. 21 presents the magnitude of the reflection coefficient $\boldsymbol{S}_{11}$.

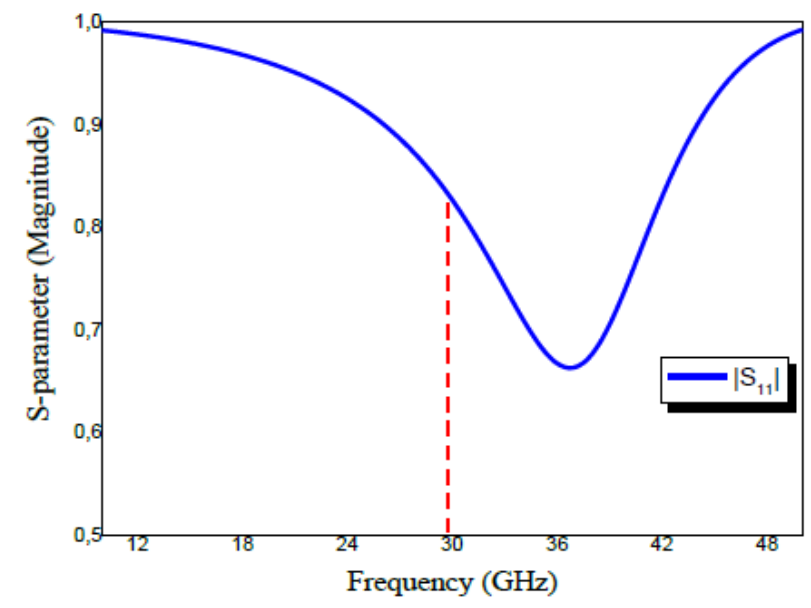

Fig. 21. Simulation of the $\boldsymbol{S}_{11}$ coefficient of the PRS unit cell (CST MW result simulation).

Fig. 22 presents the phase of the reflection coefficient $\boldsymbol{S}_{11}$.

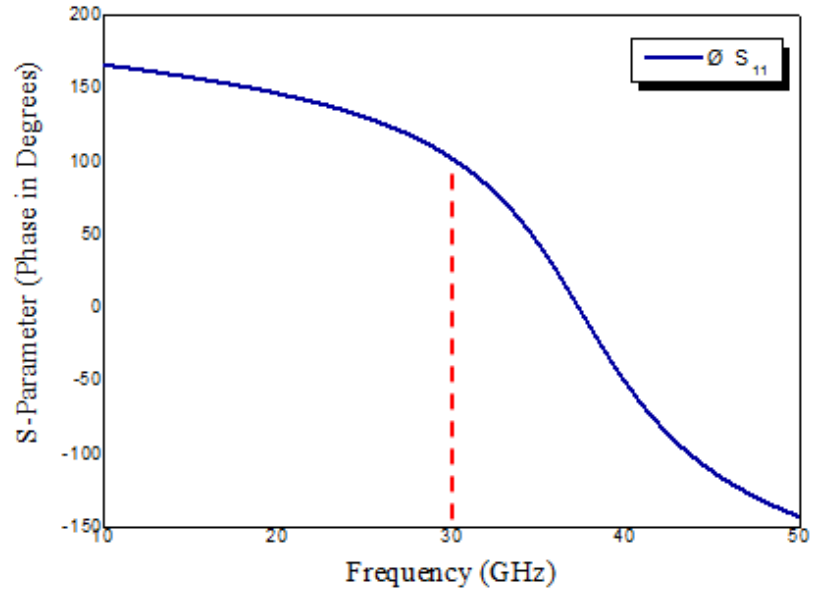

Fig. 22. Simulation of the phase of the S11 coefficient of the PRS unit cell (CST MW result simulation).

The PRS presents a resonance $\left(\Phi_{P R S}=0^{\circ}\right)$ at $37 \mathrm{GHz}$ and shows a high reflectivity with relatively low phase values $\left(<90^{\circ}\right)$ around $35 \mathrm{GHz}$ (Fig. 21). This high reflectivity is a very important parameter for obtaining a directional beam in such cavity.

According to (8), the cavity thickness that ensures a resonance frequency of $30 \mathrm{GHz}$ is $\mathrm{d}=7 \mathrm{~mm}$.

The simulation results of $\boldsymbol{S}_{11}$ coefficient and the radiation patterns of EBG antenna at the resonance are shown in Fig. 23 and 25 .

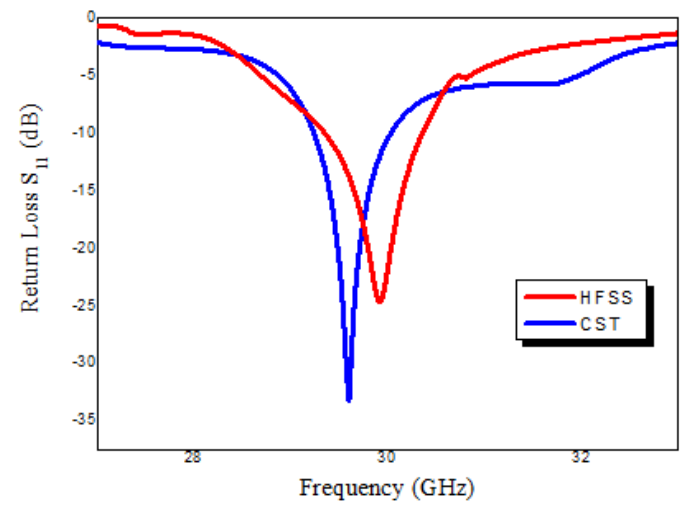

Fig. 23. Simulated reflection coefficient for the proposed PRS-AMC antenna.

The simulated bandwidth at $-10 \mathrm{~dB}$ is between 29.3 and $30.2 \mathrm{GHz}$.

Axial ratio simulation results of this antenna are shown in Fig. 24(a) and (b). 


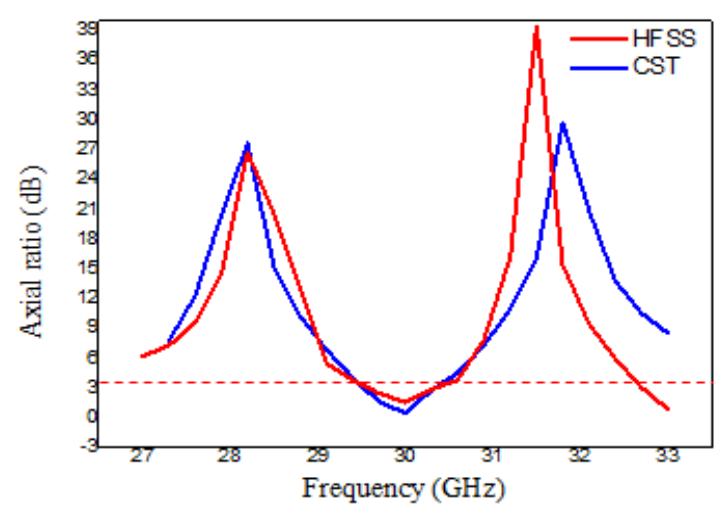

(a)

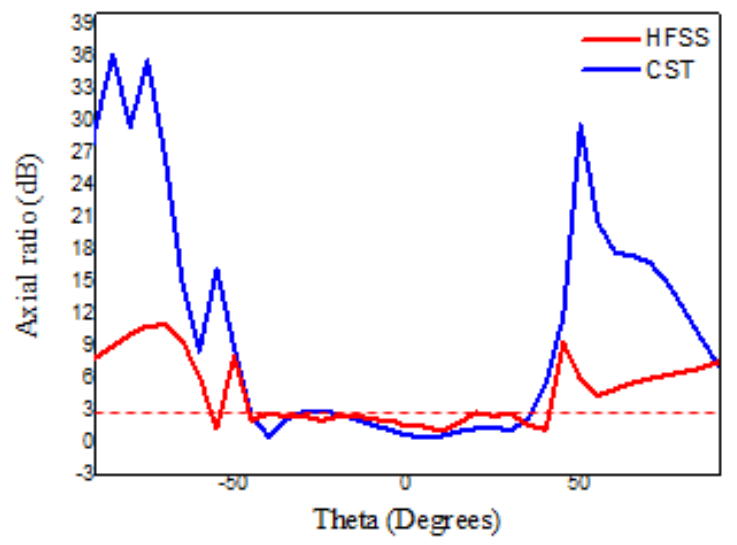

(b)

Fig. 24. Axial ratio as a function of (a) frequency, (b) elevation.

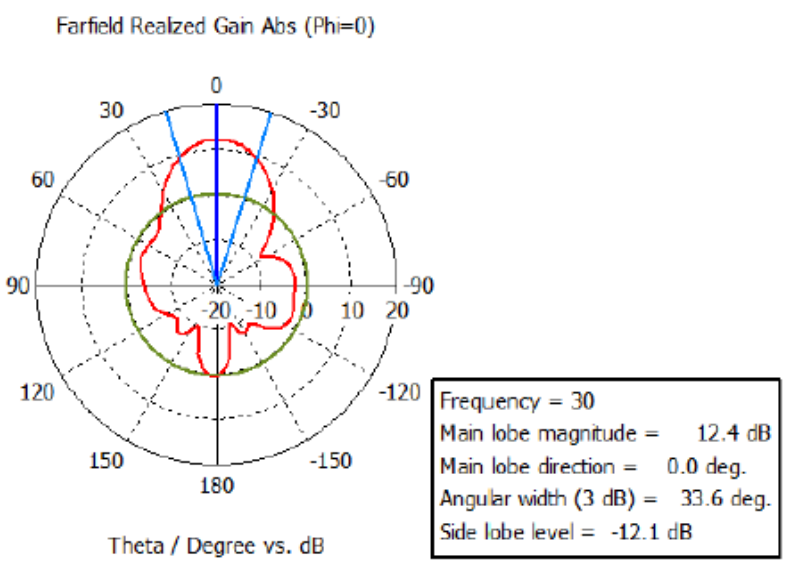

Fig. 25. Radiation pattern of proposed antenna with AMC and PRS (CST MW result simulation).

\section{RELATED WORK}

The recent development of different antennas design techniques enabled a breakthrough in many different areas of science and technology. Microstrip patch antenna has gained attraction for wide application in microwave frequencies range.

The idea of RFID millimetre wave or also called Millimetre Wave Identification (MMID) was first proposed in [41] and analysed in more detail in [42]. First, the highest frequency used for RFID was $24 \mathrm{GHz}$ per Biebl [43]. The main differences between the RFID systems and the MMID systems were the wavelength, the effective size opening of the main beam and the radar section. The MMID also allows communication at higher data rates, due to its large bandwidth, which is available for example at $30 \mathrm{GHz}$. At millimeter waves, range a small antenna can provide an important gain. However, this cannot be used to increase the gain of the tag, because this makes the transponder only accessible from the main beam direction.

The characteristics of the proposed antenna are indicated in Table 2.

TABLE II. A COMPARISON BETWEen the Proposed ANTENNA ANd Other Published MiLlimeter WAVE ANTENNAS

\begin{tabular}{|l|l|l|l|l|l|}
\hline & This work & MOTL 2015 [12] & $\begin{array}{l}\text { RADIOENGINEERING } \\
\text { [44] }\end{array}$ & $\begin{array}{l}\text { RADIOENGINEERING } \\
\text { [44] }\end{array}$ & $\begin{array}{l}\text { RADIOENGINEERING } \\
\text { [44] }\end{array}$ \\
\hline Technology & $\begin{array}{l}\text { Dual-feed Patch } \\
\text { antenna }\end{array}$ & $\begin{array}{l}\text { Encoched Patch } \\
\text { antenna }\end{array}$ & Bow-Tie Antenna & Vivaldi antenna & Spiral antenna \\
\hline frequency [GHz] & 30 & 30 & $30-40$ & $20-40$ & 40 \\
\hline Polarization & Circular & linear & linear & linear & linear \\
\hline Gain [dB] & $12.1 @ 30 \mathrm{GHz}$ & $14 @ 30 \mathrm{GHz}$ & $6.32 @ 40 \mathrm{GHz}$ & $5.89 @ 40 \mathrm{GHz}$ & $7.62 @ 40 \mathrm{GHz}$ \\
\hline Area [mm $\left.{ }^{2}\right]$ & $17 * 17$ & $36.9 * 25.3$ & $8.191 * 10.3$ & $12.71 * 15.53$ & $120 * 16$ \\
\hline
\end{tabular}

Summarized in Table 2, is a performance comparison of the proposed antenna with the various studied antennas. Compared to the conventional metallic antennas in [12] and [44], the performance of the proposed structure shows a considerable improvement. In addition, compared with other Technologies, the present work present a low-cost and a simple structured antenna not only attains comparable gain and efficiency but also is of a notably smaller size.
The major contribution of the present work is to have a circular polarization while keeping a good efficiency of the antenna.

\section{CONCLUSION}

Relevant improvement of the antennas performances using surfaces based on planar artificial magnetic conductors has been analyzed and shown in this article. A Fabry-Perot cavity is designed from these metamaterials. The performance of a 
printed antenna in terms of directivity has been increased. Despite significant losses in metamaterials, based AMC high directivity can be achieved. Moreover, the simple construction of this type of material compared to the Sievenpiper structures with the metalized holes makes it possible to reduce the manufacturing costs for this type of application.

First, the designed patch antenna was performed to obtain a high gain $(8.16 \mathrm{~dB})$ with circular polarization, then a HIS was added on its surface to enhance the gain. The new antenna obtained presents a gain of $9.1 \mathrm{~dB}$ and a side lobe level less than $-14 \mathrm{~dB}$.

Second, theoretical expressions are used to evaluate the thickness of the FP cavity and to estimate the maximum directivity of the ideal infinite antenna.

Since the directivity depends directly on the quality factor of the cavity, the latter depends on the volume of the cavity, a compromise must be found between the compactness of the structure and its efficiency. Acceptable performances are possible with very compact cavities compared to traditional structures based on Fabry-Perot or photonic band gap resonators. The last part concerning the optimization of the cavity has shown the possibility of producing structures with high directivity and ultra-compact. Indeed, by working outside the behavior area in AMC, losses can be avoided in these metamaterials to obtain a better yield of the overall structure. The role of the PRS based on AMC was therefore very important for the proper functioning of the cavity and to reach especially low thicknesses. It is therefore necessary to design it wisely in order to improve the efficiency of an antenna in terms of gain, compactness and radiance.

In conclusion, the interests of the planar AMC metamaterials use in the antennas field are multiple. They concern gain, directivity and compactness of these structures. A demonstration of some of these benefits has been presented in this paper.

\section{ACKNOWLEDGMENT}

The authors would like to thank Faculty of Science, University El Manar Tunisia and all the team of Research Unit for High Frequency Electronic Circuits and Systems for supporting this project.

\section{REFERENCES}

[1] G. KUMAR - K.P. RAY. Broadband Microstrip Antennas. Editor Artech House, Broadband Circularly Polarized MSAs, pp 309-356. 2003

[2] Jianjun Wu , Yingzeng Yin, Zedong Wang and Ruina Lian. Dual-Band Circularly Polarized Antenna with Differential Feeding. Progress In Electromagnetics Research C, Vol. 49, 11-17, 2014

[3] Zhao, Y., Z. Zhang, K. Wei, and Z. Feng. A dual circularly polarized waveguide antenna with bidirectional radiations of the same sense. IEEE Transactions on Antennas and Propagation, Vol. 62, No. 1, 480-484, 2014.

[4] Ch. Guesmi, A. Ferchichi, A. Gharsallah. A Fractal Circular Polarized RFID Tag Antenna. Cent. Eur. J. Eng. DOI: 10.2478/s13531-012-00727. 2013

[5] J. W. ZHANG - S. S. ZHONG - S. Q. XU. Compact broadband circularly polarized microstrip antenna. Microwave and Optical Technology Letters Vol. 48, Issue 9, Pages 1730 - 1732. June 2006
[6] He, Y., W. He and H. Wong. A wideband circularly polarized crossdipole antenna. IEEE Antennas Wireless Propagation Letters, Vol. 13, 67-70. 2014

[7] H. C. LIEN - Y. C. L EE - H. C. TSAI. Couple-fed Circular Polarization Bow Tie Microstrip Antenna. PIERS Online Vol. 3 No. 2 pp : 220-224. 2007

[8] Ch. Guesmi, A. Ferchichi, A. Gharsallah. A Modified Fractal Bow Tie Antenna for an RFID Reader. International Journal of Electrical and Computer Engineering (IJECE), Vol. 4, No. 3, pp. 441 446, ISSN: 2088-8708. June 2014.

[9] Deng, J., L. Guo, T. Fan, Z. Wu, Y. Hu, and J. Yang. Wideband circularly polarized suspended patch antenna with indented edge and gap-coupled feed. Progress In Electromagnetics Research, Vol. 135, 151-159, 2013.

[10] B. Subbarao and V.F. Fusco. Compact polarization select able radial cavity antenna. Electronics Letters, Vol. 41, Issue 12, Page(s): $671-$ 672. June 2005

[11] Hsieh, W.-T., T.-H. Chang and J.-F. Kiang. Dual-band circularly polarized cavity-backed annular slot antenna for GPS receiver. IEEE Transactions on Antennas and Propagation, Vol. 60, No. 4, 2076-2080, 2012.

[12] O. Necibi, D. Hamzaoui, TP. Vuong and A. Gharsallah. A Novel RFIDHIS-PRS Reader Antenna for the Millimeter Wave Band $30 \mathrm{GHz}$. Microwave and Optical Technology Letters, Vol. 57, No. 8, pp. 18351842 (DOI): $10.1002 / \mathrm{mop} .29201$. August 2015

[13] O. Necibi, D. Hamzaoui, TP. Vuong and A. Gharsallah. A Novel RFID Antenna with HIS Structure for Ka Band. International Journal on Communications Antenna and Propagation (IReCAP.), Vol. 5, No. 3. ISSN 2039 - 5086. June 2015.

[14] O. Necibi, A. Ferchichi, TP. Vuong, A. Gharsallah. Miniaturized CSRR TAG Antennas for $60 \mathrm{GHz}$ Applications. International Journal of Electrical and Computer Engineering (IJECE), Vol.3, No.5, pp. 31 41. February 2014.

[15] Deng, C., Y. Li, Z. Zhang, G. Pan and Z. Feng. Dual-band circularly polarized rotated patch antenna with a parasitic circular patch loading. IEEE Antennas Wireless Propagation Letters, Vol. 12, 492-495, 2013.

[16] Wu, H., J. Zhang, L. Yan, L. Han, R. Yang, and W. Zhang. Differential dual-band antenna-in package with T-shaped slots. IEEE Antennas Wireless Propagation Letters, Vol. 11, 1446-1449, 2012.

[17] Xue, Q., S. W. Liao and J. H. Xu. A differentially driven dual-polarized magneto-electric dipole antenna. IEEE Transactions on Antennas and Propagation, Vol. 61, No. 1, 425-430, 2013.

[18] K. T. V. REDDY - G. KUMAR. Dual-feed gap-coupled square microstrip antennas for broadband circular polarization. Journal of Microwave and Optical Letters, Vol. 26, Issue 6, Pages 399 - 402

[19] S. ASSAILLY - C. TERRET - JP. DANIEL - K. MAHDJOUBI. Low cost stacked circular polarized microstrip antenna. Symposium Antennas and Propagation Society International 1989. AP-S. Digest, 26-30 June 1989 Page(s) :628 - 631 Vol.2

[20] M. KOSSEL - H BENEDICKTER - W. BAECHTOLD. Circular polarized aperture coupled patch antennas for an RFID system in the 2.4 GHz ISM band. IEEE Radio and Wireless Conference, 1999. RAWCON 99. Page(s) :235 - 238

[21] J. W. ZHANG - S. S. ZHONG - S. Q. XU. Compact broadband circularly polarized microstrip antenna. Microwave and Optical Technology Letters Vol. 48, Issue 9, Pages 1730 - 1732.

[22] A. Balanis. Antenna theory analysis and design. Constantine A. Balanis, Arizona State University Tempe, AZ.2005.

[23] John L. Volakis: 'Antenna engineering 'HANDBOOK, University Stuttgart 2007.

[24] Huang, Y. and Boyle, K. Antennas from theory to practice. 1st edn, England: John. 2008.

[25] H.N. Bao Phuong, D.N. Chien, and T.M. Tuan. Novel design of electromagnetic band gap using fractal geometry. Hindawi Publishing Corporation, Int J Antennas Propag 2013 (2013), 1-8.

[26] A. Ghiotto, S.F. Cantalice, T.P. Vuong, A. Pouzin, G. Fontgalland, and S. Tedjini. Miniaturized patch antenna for the radio frequency 
identification of metallic objects. IEEE Antenna and Propagation Society International Symposium, San Diego, CA, 2008, pp. 1-4.

[27] Yang, F. and Y. Rahmat-Samii. Reflection phase characterizations of the EBG ground plane for low profile wire antenna applications. IEEE Transactions on Antennas and Propagation, Vol. 51, No. 10, 2003.

[28] Gonzalo, R., P. de Maagt, and M. Sorolla. Enhanced patch antenna performance by sup-pressing surface waves using photonic-bandgap substrates. IEEE Transactions on Microwave Theory and Techniques, Vol. 47, No. 11, 1999.

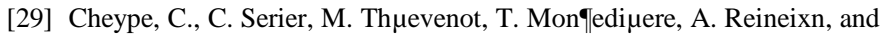
B. Jecko. An electromagnetic bandgap resonator antenna. IEEE Transactions on Antennas and Propagation, Vol. 50,No. 9, 2002.

[30] Kim, Y., F. Yang, and A. Z. Elsherbeni. Compact artificial magnetic conductor designs using planar square spiral geometries. Progress in Electromagnetics Research, PIER 77, 43\{54, 2007.

[31] D. Sievenpiper. High-Impedance Electromagnetic Surfaces. Ph.D. Dissertation, University of California, Los Angeles, 1999

[32] S. Clavijo, R. E. Diaz, and W. E. McKinzie. Design methodology for Sievenpiper high-impedance surfaces: an artificial magnetic conductor for positive gain electrically small antennas. IEEE Trans. Antennas Propag., vol. 51, no. 10, pp. 2678-2690, Oct. 2003.

[33] Y. Fu, N. Yuan and G. Zhang. Compact high-impedance surfaces incorporated with interdigital structure. ELECTRONICS LETTERS 4th March 2004 Vol. 40 No. 5

[34] Y. Rahmat-Samii. EBG Structures for Low Profile Design Antenna: What Have We Learned?. Antennas Propagation, EUCAP 2007, pp.1 5, 11-16 Nov. 2007.

[35] R. CHANTALAT. Optimisation d'un réflecteur spatial à couverture cellulaire par l'utilisation d'une antenne à bande interdite électromagnétique multi-sources. Thèse de doctorat $\mathrm{n}^{\circ} 36-2003$, U.E.R. des Sciences, Université de Limoges, novembre 2003

[36] S. WANG, A.P. FEREDISIS, G. GOUSSETIS, J.C. VARDAXOGLOU Low-Profile resonant cavity antenna with artificial magnetic conductor ground plane. Electronic Letters, Volume $40 \mathrm{n}^{\circ}$ 7, 2004.
[37] A.P. FEREDISIS, G. GOUSSETIS, S. WANG, J.C. VARDAXOGLOU. Artificial magnetic conductor surfaces and their application to low profile high gain planar antennas. IEEE Transactions on Antennas and Propagation, Volume $53 \mathrm{n}^{\circ}$ 1: 209-215, 2005.

[38] S. WANG, A.P. FEREDISIS, G. GOUSSETIS, J.C. VARDAXOGLOU. High-gain subwavelength resonant cavity antennas based on metamaterial ground plan. IEE Proceedings in Microwave, Antennas and Propagation, Volume $153 \mathrm{n}^{\circ} 1$ : 1-6, 2006.

[39] D. SIEVENPIPER, L. ZHANG, R. F. J. BROAS, N. G. ALEXOPOULOS and E. YABLONOVITCH. High-impedance electromagnetic surfaces with a forbidden frequency band. IEEE Transactions on Microwave Theory and Techniques, vol. 47, no. 11, pp. 2059-2074, 1999.

[40] L. ZHOU, H. LI, Y. QIN, Z. WEI, C.T. CHAN. Directive emissions from subwavelength metamaterial-based cavities. Applied Physics Letters, Volume 86, 2005.

[41] D. Neculoiu, G. Konstantinidis, T. V“ah"a-Heikkil“a, A. M"uller, D. Vasilache, A. Stavinidris, L. Bary, M. Dragoman, I. Petrini, C. Buiculescu, Z. Hazoupulos, N. Kornilios, P. Pursula, R. Plana And D. Dascalu. GaAs Membrane-Supported $60 \mathrm{GHz}$ Receiver with Yagi-Uda Antenna. MEMSWAVE 2007, 8th International Symposium on RF MEMS and RF Microsystems, Barcelona, Spain, June 26 - 29, 2007, pp. $15-18$.

[42] P. Pursula, T. V"ah"a-Heikkil"a, A. M"uller, D. Neculoiu, G. Konstantinidis, A. Oja, and J. Tuovinen; Millimeter-Wave Identification - A New Short-Range Radio System for Low-Power High Data-Rate Applications. IEEE Transactions on Microwave Theory and Techniques, Vol. 56, Issue 10, pp. 2221 - 2228, October 2008.

[43] E. M. Biebl. RF Systems Based on Active Integrated Antennas. (AEU) International Journal of Electronics and Communications ", Vol. 57, No. 3 , pp. $173-180,2003$.

[44] Kamil PÍTRA, Zbyněk RAIDA. Planar Millimeter-Wave Antennas: A Comparative Study. RADIOENGINEERING, VOL. 20, NO. 1, APRIL 2011. 